\title{
BMJ Open Effect of cervical manipulation on vertebral artery and cerebral haemodynamics in patients with chronic neck pain: a crossover randomised controlled trial
}

Nicholas Moser, ${ }^{1}$ Silvano Mior, ${ }^{1,2}$ Michael Noseworthy, ${ }^{3}$ Pierre Côté, ${ }^{4}$ Greg Wells, ${ }^{5}$ Michael Behr, ${ }^{6}$ John Triano ${ }^{1}$

To cite: Moser N, Mior S, Noseworthy M, et al. Effect of cervical manipulation on vertebral artery and cerebral haemodynamics in patients with chronic neck pain: a crossover randomised controlled trial. BMJ Open 2019;9:e025219. doi:10.1136/ bmjopen-2018-025219

- Prepublication history and additional material for this paper are available online. To view these files, please visit the journal online (http://dx.doi. org/10.1136/bmjopen-2018025219).

Received 6 July 2018 Revised 18 January 2019 Accepted 5 March 2019

Check for updates

(C) Author(s) (or their employer(s)) 2019. Re-use permitted under CC BY-NC. No commercial re-use. See rights and permissions. Published by BMJ.

For numbered affiliations see end of article.

Correspondence to

Dr Nicholas Moser;

nicholasvcmoser@gmail.com

\section{ABSTRACT}

Objective It is hypothesised that cervical manipulation may increase the risk of cerebrovascular accidents. We aimed to determine whether cervical spine manipulation is associated with changes in vertebral artery and cerebrovascular haemodynamics measured with MRI compared with neutral neck position and maximum neck rotation in patients with chronic neck pain.

Setting The Imaging Research Centre at St. Joseph's Hospital in Hamilton, Ontario, Canada.

Participants Twenty patients were included. The mean age was 32 years ( $S D \pm 12.5)$, mean neck pain duration was 5.3 years $(S D \pm 5.7)$ and mean neck disability index score was $13 / 50$ ( $S D \pm 6.4$ ).

Interventions Following baseline measurement of cerebrovascular haemodynamics, we randomised participants to: (1) maximal neck rotation followed by cervical manipulation or (2) cervical manipulation followed by maximal neck rotation. The primary outcome, vertebral arteries and cerebral haemodynamics, was measured after each intervention and was obtained by measuring three-dimensional T1-weighted high-resolution anatomical images, arterial spin labelling and phase-contrast flow encoded MRI. Our secondary outcome was functional connectivity within the default mode network measured with resting state functional MRI.

Results Compared with neutral neck position, we found a significant change in contralateral blood flow following maximal neck rotation. There was also a significant change in contralateral vertebral artery blood velocity following maximal neck rotation and cervical manipulation. We found no significant changes within the cerebral haemodynamics following cervical manipulation or maximal neck rotation. However, we observed significant increases in functional connectivity in the posterior cerebrum and cerebellum (resting state MRI) after manipulation and maximum rotation.

Conclusion Our results are in accordance with previous work, which has shown a decrease in blood flow and velocity in the contralateral vertebral artery with head rotation. This may explain why we also observed a decrease in blood velocity with manipulation because it involves neck rotation. Our work is the first to show that cervical manipulation does not result in brain perfusion
Strengths and limitations of this study

- A strength of the study was the design, which ensured control of confounders and provided statistical efficiency.

- We used what is considered the criterion standard for both diagnosis of vertebrobasilar artery stroke and quantifying blood flow (phase-contrast MRI) because of its greater sensitivity compared with ultrasonography.

- A limitation of the study was the restriction of analysis to a time following the test manoeuvres. Realtime measures currently are technically not feasible and transient effects of various neck positions on vertebral artery and cerebrovascular haemodynamics may have been missed.

changes compared with a neutral neck position or maximal neck rotation. The changes observed were found to not be clinically meaningful and suggests that cervical manipulation may not increase the risk of cerebrovascular events through a haemodynamic mechanism.

Trial registration number NCT02667821

\section{INTRODUCTION}

Anecdotal evidence from case reports and case series suggest that neck manipulation increases the risk of vertebrobasilar artery stroke. $^{1-3}$ However, the epidemiological evidence does not support this hypothesis. ${ }^{45}$ In their case-crossover study, Cassidy et al found that the risk of vertebrobasilar artery stroke was similar for patients with neck pain or headaches who consult physicians and those who consult chiropractors. ${ }^{4}$ This suggests that the hypothesised association is due to protopathic bias.

Understanding whether neck manipulation increases the risk of stroke is important because patients with neck pain frequently 
consult chiropractors and manipulation of the cervical spine is commonly performed for symptomatic relief. ${ }^{6-10}$ Dissection of the vertebral artery is involved in most cases that implicate cervical manipulation. ${ }^{11}$ However, when damage to the vertebral artery is absent, vasospasm ${ }^{12-15}$ and 'subclinical' endothelial injury have also been hypothesised to be causes of stroke. According to the vasospasm hypothesis, placing the head in rotation and hyperextension during a manipulation leads to considerable stress and stretch forces in the vertebral artery, specifically at the $\mathrm{C} 2 / \mathrm{C} 1$ and cephalad/distal portion of the vertebral artery. ${ }^{16-18}$ This mechanical compression or stretching of the vertebral artery may lead to changes in blood flow and the subclinical injury to the vertebral artery can lead to thrombosis. $^{19}$

Several studies have investigated changes in blood flow during cervical spine motion. ${ }^{20-28}$ Most studies report a decrease in vertebral artery flow contralateral to the side of rotation. ${ }^{20-2325-28}$ Less is understood about blood flow during and after a cervical manipulation, but two studies found no significant changes in vertebral artery blood flow or blood velocity following cervical manipulation in healthy individuals. ${ }^{29} 30$ However, the impact of cervical manipulation on vertebral artery blood flow in the population likely to undergo this manoeuvre for therapeutic purposes is unknown. Neck pain patients may differ from that of healthy populations because moderate to severe perceived neck disability, as measured with the Neck Disability Index (NDI) is correlated with cortical hypoperfusion. ${ }^{31}$

Therefore, our primary aim was to determine whether cervical manipulation leads to a meaningful change in vertebral and cerebral haemodynamics compared with neutral neck position or neck rotation in adult patients with chronic neck pain. Our secondary aim was to compare the functional connectivity within the default mode network (DMN) between neutral neck position, neck rotation and cervical manipulation. We hypothesised that cervical manipulation or maximal neck rotation is associated with significant change in vertebral and cerebral haemodynamics compared with neutral neck position in adult patients with chronic neck pain.

\section{METHODS \\ Study design}

We conducted a crossover randomised controlled study. In each subject, we randomised the sequence of cervical manipulation and maximal neck rotation and compared their effects on cerebrovascular haemodynamics. No washout period was used between each intervention. It was assumed that the time needed to measure the blood haemodynamics allowed enough time to return to their baseline status. $^{32}$

\section{Participants}

Patients who were eligible for the study were attending a teaching clinic of the Canadian Memorial Chiropractic
College, Toronto, Canada between September 2016 and April 2017. We recruited participants via poster advertising displayed at the teaching clinic, word of mouth and referrals from the supervising clinicians at the clinic. To be included, patients had to meet the following criteria: (1) at least 18 years old; (2) chronic neck pain ( $\geq 3$ months' duration) defined as either neck pain-associated disorder or whiplash-associated disorder; (3) grade I-II neck pain, ${ }^{33}$ defined as neck pain with no signs or symptoms of major structural pathology, which may or may not interfere with activities of daily living; (4) prescribed cervical manipulation by the clinician at the teaching clinic supervising their care and (5) provide written informed consent. Exclusion criteria were a history of neck pain with associated arm pain within the last 6 months; any current or history of neurological symptoms including facial or extremity weakness, abnormal sensation to the face, body, or extremities, uncontrolled movements, abnormal gait, dizziness, unexplained nausea/vomiting, difficulty with speaking or swallowing; history of new or severe (Visual Analogue Scale $>6 / 10$ ) headaches in the last 3 months; any contraindications to MRI; or any history of using drugs that affect blood flow such as warfarin, or anticoagulants. In addition, all participants refrained from vigorous physical activity and ingesting alcohol and caffeine 1 day before their scheduled participation.

\section{Randomisation and masking}

We used simple randomisation to allocate participants to one of two sequences of interventions: (1) maximal neck rotation followed by upper cervical manipulation or (2) upper cervical manipulation followed by maximal neck rotation. The study coordinator (NM) conducted the randomisation using a randomised table generator (GraphPad Software, La Jolla, California, USA). The random allocation was communicated verbally to the study clinician (SM) on the day of the study protocol. Randomisation was concealed, no other study personnel or participants were aware of the intervention assignments.

\section{Procedures}

Prior to commencement of the study protocol, participants underwent a cervical spine examination by the clinician (SM) performing the test manoeuvres to identify the site of manipulation. Baseline information on each participant was collected and included: age, gender, height, weight, NDI score, neck pain intensity, duration of neck complaint and headache pain intensity (table 1).

Baseline MRI of the upper cervical spine and brain with the neck in the neutral position was conducted before randomisation. Neutral neck position was defined by alignment of the Frankfort plane in a vertical orientation. For continuity of neutral alignment during imaging between test conditions, the MRI laser land-marking tool was used to triangulate between three oil-based markers (vitamin $\mathrm{E}$ capsules) taped to the nasion (bridge of the nose) and immediately in front of the tragus of the ears, bilaterally. 


\begin{tabular}{lc}
\multicolumn{2}{l}{ Table 1 Baseline participant characteristics } \\
\hline Participant characteristics & Mean (SD) \\
\hline Age (years) & $32.05(12.5)$ \\
Sex, n (\%) & $14(70 \%)$ \\
$\quad$ Female & $169(8.9)$ \\
Height (cm) & $69.6(18.7)$ \\
Weight (kg) & $13(6.4)$ \\
NDI & $4.7(2.1)$ \\
NRS neck & $5.3(5.7)$ \\
Duration of complaint (years) & $3(2.6)$ \\
NRS headache &
\end{tabular}

NDI, Neck Disability Index; NRS, numerical rating scale.

Following random allocation, either maximal neck rotation or cervical manipulation was first performed, followed by the other procedure. Maximal neck rotation was achieved by instructing participants to rotate their head as far as comfortably possible in the direction opposite to the side of clinical symptoms as elicited during the cervical spine examination. The clinician performing the interventions assisted the rotation via a soft hand contact on the patient's head. The degree of maximal neck rotation was measured by an inclinometer and the position was held for $1 \mathrm{~min}$ before returning to neutral neck position for MRI sequencing. The cervical manipulation procedure was a high velocity, low amplitude impulse, with targeted contact at C1-C2 on the side of most discomfort as elicited on palpation, with the participant's head in combined axial rotation, flexion and lateral flexion postures. Variations of head positions between operators for this procedure have been demonstrated to be relatively small. ${ }^{345}$ A practitioner with $>30$ years of practice experience conducted the cervical manipulation (SM).$^{36-38}$ The manipulation procedure was performed on the adjustable and pivotal MRI bed in the MRI room with the participant in the supine position. The clinician performed the procedure by first establishing the end range of motion to determine appropriate preload position for the manipulation before applying a clinical force impulse in the coronal plane with minimal traction component.

Before each manoeuvre, the participants were queried on their comfort, condition and willingness to continue. The participant's head was repositioned to neutral immediately after each manoeuvre, and then retracted into the MRI bore. Each manoeuvre was carried out on the scanner bed in the MRI room.

\section{Outcomes}

The primary outcome measure was cerebrovascular haemodynamics within the vertebral arteries and posterior cerebrum measured with MRI. The MRI data were acquired using a 3Telsa MR750 scanner and 20-channel neurovascular array radiofrequency coil (GE Healthcare, Milwaukee, Wisconsin, USA).

The primary outcome was measured following a standardised protocol. First, the baseline MRI was performed. The head was immobilised with sponges in a neutral neck position and a localiser scan was completed. Next, high-resolution anatomical images were collected using a three-dimensional (3D) inversion recovery prepped fast-spoiled gradient recalled echo T1-weighted scan (repetition time $[\mathrm{TR}] /$ echo time $[\mathrm{TE}]=11.4 / 4.3 \mathrm{~ms}, \mathrm{TI}=450 \mathrm{~ms}$, flip angle $=12^{\circ}, 512 \times 256$ matrix, 140 slices, $24 \mathrm{~cm}$ field of view [FOV], reconstructed to $1 \mathrm{~mm}^{3}$ isotropic voxels). Following the anatomical scan, microvascular perfusion was assessed using a 3D spiral-based fast-spin echo pseudo-continuous arterial spin labelling (TE/TR/TI=10.5/4629/1525 ms, FOV $=24 \mathrm{~cm}, 512 \times 8$ spiral interleaves, $3 \mathrm{NEX}$, reconstructed to give an in-plane resolution of $1.875 \times 1.875$ [128 $\times 128]$ with $4 \mathrm{~mm}$ thick slices, scan time $=30 \mathrm{~s}$ ). Following arterial spin labelling (ASL), a phase-contrast scan for vertebral artery flow quantification was performed. At the upper cervical level C1-C2, the contralateral and ipsilateral vertebral arteries, defined to the direction of head motion, were assessed and anatomical images were established to localise the vertebral artery circulation. As previously published by Ho $e t a l,{ }^{39}$ the method for obtaining flow quantification of the vertebral artery was a two-dimensional phase-contrast pulse sequence. To capture accurate vertebral artery flow, the imaging plane is ideally perpendicular to the central axis of the blood vessel. This imaging plane was selected on the vessel of interest at the C1-C2 intervertebral level based on arterial visualisation on a maximum intensity projection of a 3D time-of-flight MRI angiogram. Acquisition parameters were as follows: fast gradient-recalled echo; echo time $/$ repeat time $=3.9 / 8.9 \mathrm{~ms}$, flip angle $=20^{\circ}$, $20 \mathrm{~cm}$ FOV; $512 \times 512$ matrix; $244 \mathrm{~Hz} /$ pixel receiver bandwidth; 1 average; $4 \mathrm{~mm}$ thick; and velocity encoding of 50 $\mathrm{cm} / \mathrm{s}$ encoded over 30 phases per cardiac cycle. All image measurements were obtained by manually selecting the optimal anatomical site between the base of the odontoid process and $\sim 1 \mathrm{~cm}$ above the tip of the dens. Data acquisition was triggered by peripheral gating using a pulse oximeter, with sequence acquisition time for each flow measurement being $\sim 1.5$ min, depending on heart rate. ${ }^{39}$ According to Lotz et al, vessel obliquity is tolerable to $\pm 15^{\circ}$, above which will cause a significant deviation from true flow. $^{40}$

The secondary outcome measure was functional connectivity within the DMN. For baseline measurement, this was performed immediately following the phase-contrast MRI, but prior to manipulation. Resting state functional MRI data (ie, blood oxygen level dependent, BOLD signal) was acquired using a gradient echo, echo planar imaging sequence $(64 \times 64$ matrix, 28 axial slices [ $5 \mathrm{~mm}$ thick, no skip], $24 \mathrm{~cm}$ FOV, TE/TR/flip angle $=35 \mathrm{~ms} / 2000 \mathrm{~ms} / 90^{\circ}, 180$ temporal points, total scan time $=6 \mathrm{~min})$. During the resting state scan, participants were asked to keep their eyes open, stay awake and not think of anything in particular. 
Following the baseline MRI scan, the image sequence to obtain the primary and secondary outcomes changed to the following: 3-plane localiser, ASL, phase-contrast, resting state MRI and 3D anatomical scan. The latter three were always performed with the $3 \mathrm{D}$ anatomical last seeing as microvascular changes (ie, blood flow) were more likely to be detected early after the cervical manipulation and maximal neck rotation, while structural changes (ie, 3D scan) would not be expected to occur. Participants were scanned a total of three times for each session: baseline, and two procedures in random order.

After testing, each participant was observed for 1 hour and contacted by a study coordinator (NM) within 24 hours of release for follow-up on status. Adverse events, defined as side effects that are harmful, were assessed via open-ended questions. ${ }^{41}$ These included local soreness and pain in the area of the applied test manoeuvres (minor adverse events), as well as signs of stroke or transient ischaemic attack (major adverse events).

\section{Statistical analysis}

Previous research examining reproducibility of cerebral perfusion measurements using ASL suggested that the mean per cent perfusion difference was $7.1(\mathrm{SD} \pm 12){ }^{42}$ To date, no study has provided information on cerebral blood flow and perfusion after mechanical challenges to the cervical spine. ${ }^{43}$ Since minimal clinically important differences have not yet been established, we chose to calculate the study sample size by assuming an effect size. We assumed that a change of at least $2 \mathrm{SD}$ from the normal mean flow would indicate a significant variability of the haemodynamics to the mechanical challenges performed to the cervical spine. Based on this effect size, a power of $80 \%$, a significance level at $\mathrm{p}<0.05$ and two-sided t-test, we calculated that a sample size of 16 participants was necessary. ${ }^{43}$ We increased our sample size by $20 \%$ ( $n=20$ participants) to account for possible dropouts.

An experienced analyst (MB), who remained blinded to the sequence of test manoeuvres, performed the data quantification of the primary and secondary outcomes. Microvascular perfusion and resting state MRI data were analysed using analysis of functional neuroimaging (AFNI). ${ }^{44}$ For each participant, all ASL and resting state MRI data were spatially registered to the initial (neutral condition) position. Anatomical, blood flow and functional data were transformed automatically to the Colin27 atlas, using the AFNI command @auto_tlrc, with functional data resampled to a $2 \mathrm{~mm}$ isometric grid. $^{45}$ Temporal band-pass filtering with cut-offs of $0.009 \mathrm{~Hz}<\mathrm{f}$ $<0.08 \mathrm{~Hz}$ was performed in order to suppress unwanted physiological signals and some hardware noise. ${ }^{46}$ Functional connectivity within the DMN was assessed using the AFNI plugin InstaCorr, a seed-based approach, which uses the Pearson method of correlation to compare time signals. ${ }^{47}$ The DMN is the most dominant temporally correlated resting network in the awake brain, defined as regions positively correlated in time with the posterior cingulate cortex (PCC) seed voxel. The PCC was defined automatically using the AFNI Talairach method 'Talairach to' and selecting a single voxel from each the left and right PCC for one analysis (ColinN27 coordinates: 10, $54,14$ and $-10,54,14)$. These were both subsequently fused in the post processing. A $5 \mathrm{~mm}$ with full width at half maximum (FWHM) Gaussian spatial smoothing filter was applied for maximise likelihood of overlap with interparticipant group analysis. Also, temporal outliers determined with the AFNI function 3dToutcount were censored out. Finally, the AFNI plugin 3dClustSim was used to threshold any clusters with fewer than 20 voxels. The ASL cerebral blood flow data were analysed similar to resting state MRI in that following spatial coregistration to the neutral condition and spatial blurring with a $5 \mathrm{~mm}$ FWHM Gaussian convolution kernel, ASL data were warped to the N27 atlas. Group analysis was accomplished using a repeat one-way within-participant 3D-analysis of variance (ANOVA), with the one factor being neck position. Post hoc testing included contrasts between neutral, maximum voluntary rotation and cervical manipulation, and also a contrast between maximum voluntary rotation and cervical manipulation. Statistical significance was defined as anything lower than an alpha value of 0.05 , with prior cluster thresholding dealing with multiple comparisons.

Flow analysis was performed using Segment V.1.9 software 47 (Medviso, Lund, Sweden). Dynamic regions of interest were drawn on the left and right vertebral arteries to quantify the mean, as well as peak velocity and flow. Data in the trigger window portion of the cardiac cycle were derived by spline interpolation using Matlab (Mathworks, Natick, Massachusetts, USA). Mean and SDs were calculated for vertebral artery blood velocity, flow, peak velocity and peak flow for each of the head conditions and vertebral artery side. Differences between task manoeuvres and vertebral artery flow and velocity were evaluated using a two-way ANOVA with factors for participants and head position. The level of significance was set at 0.05. Analyses were conducted using R-project V.2.12.1 (R Development Core Team, 2010. R: A language and environment for statistical computing. R Foundation for Statistical Computing, Vienna, Austria. ISBN 3-90005107-0, URL http:/ /www.R-project.org/) and SAS software. Vertebral artery blood flow variability was calculated over the 30 phases per cardiac cycle by examining the flow errors in the left and right vertebral arteries for each of the conditions. Additionally, we analysed the impact of order of procedures by examining the interaction between order and head position in the two-way ANOVA. ${ }^{48}$

\section{RESULTS}

Between September 2016 and April 2017, a total of 936 participants were screened for the study; 916 failed prescreening (figure 1). Most did not meet the inclusion criteria $(\mathrm{n}=890) ; 446$ participants were found to have acute neck pain (<3 months of duration); 119 participants had pain in the upper extremity and/or were identified 


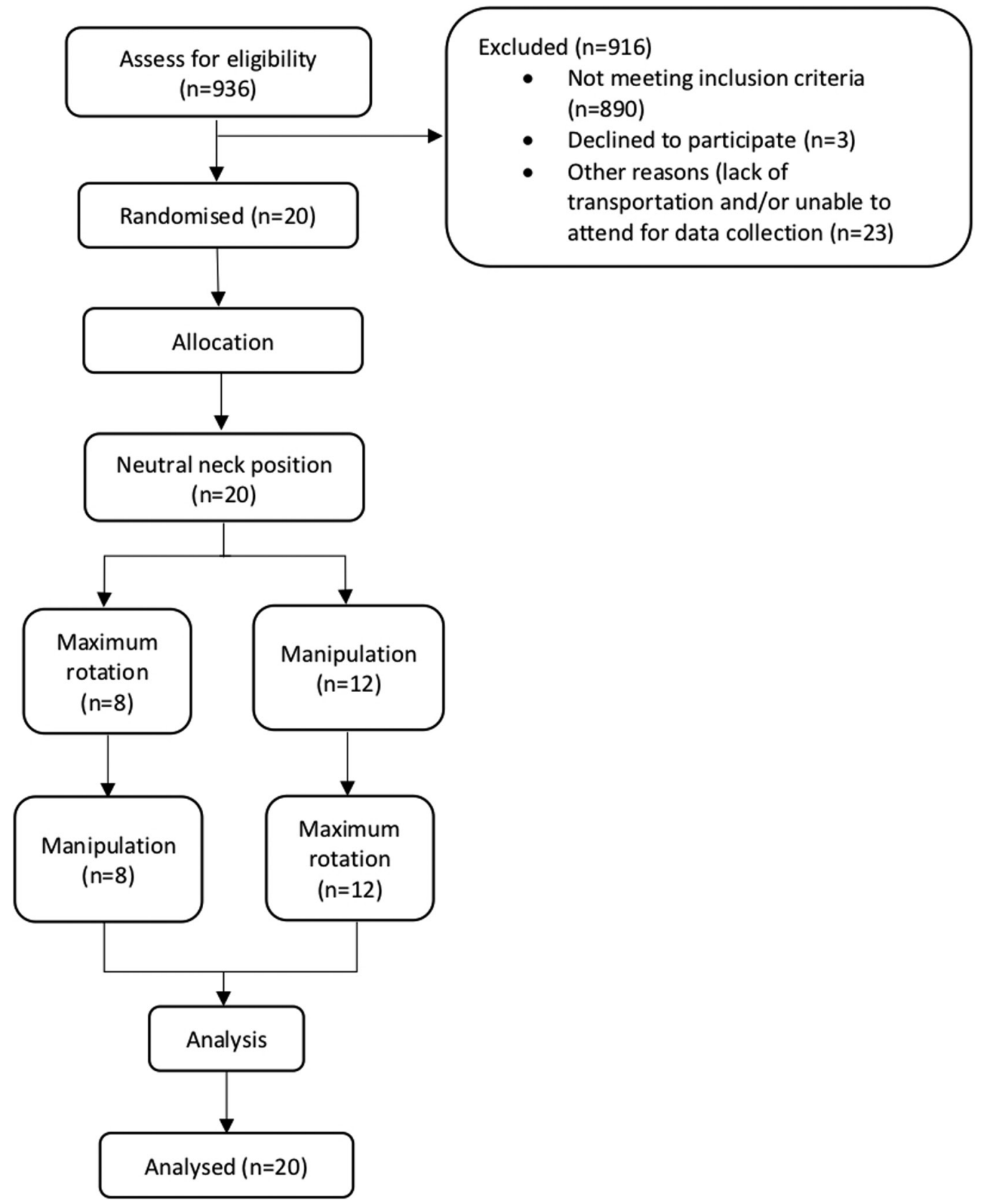

Figure 1 Protocol flow and method process.

to have radicular symptoms; 216 participants were not receiving spinal manipulative therapy to the cervical spine as part of their ongoing treatment; 68 participants reported $>6 / 10$ headache intensity on average; 15 participants were using anticoagulants; 3 participants declined to participate; 23 participants were either unable to acquire transportation to the study location or unable to attend the scheduled time. Twenty participants (14 female, 6 male) aged 23-66 years (mean [SD], 32 [ \pm 12.5$]$ years) were enrolled in the study. The average NDI score was $13 / 50(\mathrm{SD} \pm 6.4)$ with an average neck pain intensity of $5 / 10(\mathrm{SD} \pm 2.1)$ and a mean duration of neck pain of 5.3 years $(\mathrm{SD} \pm 5.7)$ (table 1$)$.

The total time elapsed for each participant testing protocol was $60 \mathrm{~min}$. The total time elapsed for each test manoeuvre was 20 min. This included applying the test manoeuvre (maximum of $1 \mathrm{~min}$ ), replacing the participant back into the MRI bore $(\sim 1 \mathrm{~min})$ and image sequencing as noted above ( 18 $\mathrm{min})$.

When compared with neutral neck position, maximal neck rotation and cervical manipulation did not significantly alter cerebral perfusion within the posterior cerebrum or cerebellum (table 2). A significant change was found in both contralateral vertebral artery blood flow and blood velocity between the three procedures (table 3). When comparing interventions to discern which interventions were different, we found a significant difference in the contralateral vertebral artery blood flow between neutral and maximal neck rotation $(0.26 \mathrm{~mL} /$ min, $95 \%$ CI 0.11 to 0.41 ) as well as between cervical 


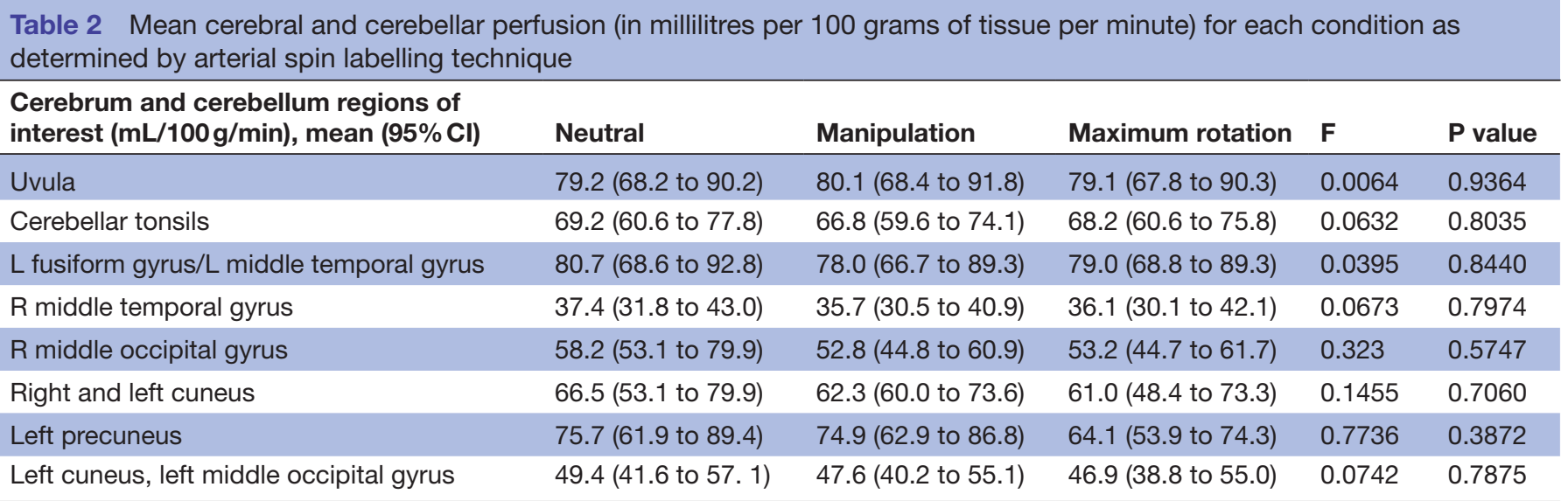

manipulation and maximal neck rotation $(0.23,95 \% \mathrm{CI}$ 0.04 to 0.42 ). We also found a significant difference in contralateral vertebral artery blood velocity between neutral and cervical manipulation $(1.15,95 \%$ CI 0.4 to 1.9) and between neutral and maximal neck rotation $(1.18,95 \%$ CI 0.77 to 1.59$)$ (table 4). Finally, we found no significant differences in flow variability within the vertebral arteries between the interventions (online supplementary figures I and II).

We measured a significant increased functional connectivity post manipulation $(p<0.05)$. More specifically, when compared with the resting position, the following areas showed significantly increased functional connectivity: uvula, cerebellar tonsils, left fusiform gyrus and left middle temporal gyrus, right middle temporal gyrus, right middle occipital gyrus, bilateral cuneus, left precuneus and left middle occipital gyrus (online supplementary table I).

When examining for an order effect of experimental procedures on outcomes, we found a significant order effect only for contralateral vertebral artery blood velocity (table $5, \mathrm{p}=0.02$ ). For subjects receiving the manipulation first, the difference in contralateral velocity between manipulation and maximal rotation conditions is on average $0.50 \mathrm{~cm} / \mathrm{s}(95 \% \mathrm{CI}-0.15$ to 1.16$)$, whereas it is on average $-0.60 \mathrm{~cm} / \mathrm{s}(95 \% \mathrm{CI}-1.54$ to 0.34$)$ if they received the manipulation second. That is, velocity is higher for which ever condition comes first.
No major adverse events were reported. One minor adverse event, mild neck soreness, was reported immediately post procedure by one participant. The participant attributed the soreness to having to lay motionless on the hard scanner bed during study protocol.

\section{DISCUSSION}

Our primary objective was to assess the cerebrovascular and vertebral artery blood flow and velocity changes between various head positions including cervical manipulation in patients with chronic neck pain. We found no significant cerebral perfusion changes within the posterior cerebrum or cerebellum. There was, however, a significant change in the contralateral vertebral artery blood flow following maximal neck rotation. We found similar changes in the contralateral vertebral artery blood velocity following both cervical manipulation and maximal neck rotation. We found no significant change in blood flow variability between the vertebral arteries, nor between the various head positions. The decrease in contralateral blood flow to the side of maximal rotation supports previous studies. ${ }^{16}$ The finding of non-significant change in blood flow ipsilateral and contralateral to the side of cervical manipulation is also consistent with previous works. ${ }^{29}{ }^{30}$ Unlike previous work, our results suggest a significant change in contralateral blood velocity following cervical manipulation and

Table 3 ANOVA statistics comparing mean flow and velocity across head positions for ipsilateral and contralateral side, controlling for subject

\begin{tabular}{|c|c|c|c|c|c|}
\hline & $\begin{array}{l}\text { Neutral, } \\
\text { mean (SD) }\end{array}$ & $\begin{array}{l}\text { Cervical manipulation, } \\
\text { mean (SD) }\end{array}$ & $\begin{array}{l}\text { Max rotation, } \\
\text { mean (SD) }\end{array}$ & $\mathbf{F}_{2,26^{*}}$ & $P$ value \\
\hline Velocity $(\mathrm{cm} / \mathrm{s})$ ipsilateral & $15.6(3.6)$ & $14.7(3.6)$ & $15.2(3.9)$ & 2.43 & 0.11 \\
\hline Flow (mL/s) ipsilateral & $1.85(0.76)$ & $1.85(0.80)$ & $1.77(0.62)$ & 0.50 & 0.61 \\
\hline Flow (mL/s) contralateral & $1.88(0.86)$ & $1.85(0.82)$ & $1.62(0.76)$ & 6.94 & 0.004 \\
\hline
\end{tabular}

${ }^{*} \mathrm{~F}$ test from two-way ANOVA including subject and condition as factors.

ANOVA, analysis of variance. 


\begin{tabular}{lccc}
\hline & $\begin{array}{l}\text { Neutral-cervical } \\
\text { manipulation, } \\
\text { mean }(\mathbf{9 5 \%} \mathbf{C l})\end{array}$ & $\begin{array}{l}\text { Neutral-max rotation, } \\
\text { mean }(\mathbf{9 5 \%} \mathbf{~ C l})\end{array}$ & $\begin{array}{l}\text { Cervical manipulation }- \\
\text { max rotation, } \\
\text { mean }(\mathbf{9 5 \%} \mathbf{~ C l})\end{array}$ \\
\hline Velocity $(\mathrm{cm} / \mathrm{s})$ ipsilateral & $0.85(0.05$ to 1.66$)$ & $0.41(-0.37$ to 1.18$)$ & $-0.45(-1.36$ to 0.47$)$ \\
Velocity $(\mathrm{cm} / \mathrm{s})$ contralateral & $1.15(0.40$ to 1.90$)$ & $1.18(0.77$ to 1.59$)$ & $0.03(-0.53$ to 0.59$)$ \\
Flow $(\mathrm{mL} / \mathrm{s})$ ipsilateral & $-0.003(-0.18$ to 0.17$)$ & $0.07(-0.11$ to 0.26$)$ & $0.08(-0.13$ to 0.28$)$ \\
Flow $(\mathrm{mL} / \mathrm{s})$ contralateral & $0.03(-0.13$ to 0.19$)$ & $0.26(0.11$ to 0.41$)$ & $0.23(0.04$ to 0.42$)$ \\
\hline
\end{tabular}

maximal head rotation. Given the changes in vertebral artery haemodynamics are more pronounced following maximal head rotation compared with cervical manipulation, specifically in contralateral flow, the changes may be the result of the head turning rather than the effect of the thrust associated with cervical manipulation. This assumption is supported by Herzog et al who suggest that cervical manipulation imposes less stretch to the vertebral artery than the turning of the head. ${ }^{18}$

No reference values for minimally clinically important differences in the vertebral artery blood flow and velocities have been established. However, haemodynamic stenosis has been long considered as a diameter reduction of $>50 \%$, which in the vertebral artery has been associated with a peak and end-diastolic flow $>108$ and $36 \mathrm{~cm} / \mathrm{s}$, respectively. ${ }^{49}$ As suggested by Licht $e t a l^{21}$ a change in peak velocity of $>25 \%$ from baseline would be necessary for clinically relevant decrease in the vertebral artery. We observed no such reductions in our study. Furthermore, an arbitrary threshold of $200 \mathrm{~mL} / \mathrm{min}$ net vertebral artery flow volume was originally proposed and below this value patients were said to be at risk of becoming symptomatic with vertebrobasilar ischaemia. ${ }^{50}$ Seidel et $a l$, however, reported that net vertebral artery blood flow volume of less than $\sim 100 \mathrm{~mL} / \mathrm{min}$ can be considered as an indicator of low blood volume. ${ }^{51}$ In our study, the net vertebral artery blood flow volume showed that in both experimental procedures, values remained $>200 \mathrm{~mL} / \mathrm{min}$ $(222 \mathrm{~mL} / \mathrm{min}$ for cervical manipulation and $203 \mathrm{~mL} /$ min for maximal neck rotation). When examining flow changes, the largest change was $14 \%$, which occurred in the contralateral vertebral artery following maximal rotation. When we examined the vertebral artery blood velocities, we found a $7 \%$ change for both cervical manipulation and maximal rotation compared with neutral. Therefore, the relative blood flow and velocity changes observed are small and not considered clinically relevant. Continuing, none of the participants during any of the experimental procedures reported, or were observed by the investigators, to have any signs or symptoms of neurological compromise. Although vertebral artery blood flow and velocity reductions can occur with head positional changes, the individual typically remains asymptomatic due to several factors, including the presence of collateral circulation. ${ }^{52}{ }^{53}$ In the present work, this was illustrated by the preservation of cerebral perfusion despite the changes in contralateral vertebral artery haemodynamics.

Vertebrobasilar artery stroke secondary to vertebral artery dissection is a rare but devastating occurrence. ${ }^{54}$ A unique characteristic of these strokes is that they can develop in healthy adults and they frequently occur in close temporal relationship to benign neck movements, ${ }^{55}{ }^{56}$ cervical manipulation ${ }^{57}$ or trivial trauma. ${ }^{58} 59$ Due to the rarity of the condition, very little is known about the risk factors for vertebrobasilar artery stroke. Our study extends the understanding on the effects of cervical manipulation on vertebral artery and cerebrovascular haemodynamics. It is also the first study to directly measure the impact of cervical spinal manipulation on intracranial and extracranial blood flow in a chronic neck pain population. Together with previous work, ${ }^{29} 30$ our results support the position that the association between cervical manipulation and stroke is due to protopathic bias. $^{4}$

When compared with a neutral neck position, both maximum voluntary neck rotation and cervical manipulation resulted in significantly increased functional connectivity throughout the DMN as seen on the BOLD signal. Changes in the BOLD signal arise from complex interactive modulation of blood flow, blood volume and local metabolic rate, all leading to change in the local ratio of oxyhaemoglobin to deoxyhaemoglobin. This ratio drives

Table 5 Order effect on vertebral artery haemodynamics

\begin{tabular}{|c|c|c|c|c|}
\hline & $\begin{array}{l}\text { Order by position } \\
\text { interaction }\end{array}$ & $\begin{array}{l}\text { Neutral-cervical } \\
\text { manipulation }\end{array}$ & $\begin{array}{l}\text { Neutral-max } \\
\text { rotation }\end{array}$ & $\begin{array}{l}\text { Cervical manipulation - } \\
\text { max rotation }\end{array}$ \\
\hline & $F_{2,24}, p$ value & $t_{12}, p$ value & $t_{12}, p$ value & $t_{12}, p$ value \\
\hline Velocity $(\mathrm{cm} / \mathrm{s})$ contralateral & $4.33,0.02$ & $-2.23,0.045$ & $-0.69,0.51$ & $2.47,0.030$ \\
\hline Flow (mL/s) ipsilateral & $0.11,0.90$ & $-0.03,0.98$ & $0.40,0.70$ & $0.39,0.70$ \\
\hline
\end{tabular}


the change in BOLD signal through magnetic susceptibility differences that exist between oxidation states of the haemoglobin complex. The areas affected within the DMN have been identified as being involved with functions of visually guided eye movements ${ }^{60}$ facial and word recognition ${ }^{61}$ visuospatial processing ${ }^{62}$ episodic memory, reflection on self and consciousness. ${ }^{63}$ The observed increase in functional connectivity may be a consequence of sensory stimulation and patient self-awareness from handling of a body region such as the neck. When examining the aforementioned areas specifically for changes in blood flow using ASL, no significant changes were observed between any of the test head conditions. This strongly suggests that the observed increased functional connectivity were not a result of altered blood flow but secondary to changes in either blood volume or metabolic activity.

Our study had strengths. First, our design ensured control of confounders and provided statistical efficiency. Second, we used phase-contrast MRI blood flow measurement because of its greater sensitivity compared with ultrasonography (which would not have been able to adequately penetrate through bone to probe the vessels we wanted to measure), and because it is considered the criterion standard for both diagnosis of vertebrobasilar artery stroke and quantifying blood flow. ${ }^{394-67}$ Estimates of repeatability of flow measures were made in preliminary work by quantifying vertebral artery flow in a single healthy participant twice over a 2-month interval. ${ }^{29}$

A limitation of the study was the restriction of analysis to a time (average 115s) following the test manoeuvres. Real-time measures currently are technically not feasible and transient effects of various neck positions on vertebral artery and cerebrovascular haemodynamics may have been missed. Post manoeuvre analysis makes comparisons with other real-time studies challenging. Thus, the results only describe post procedural effects and cannot be generalised to the possible effects occurring during the test manoeuvres. However, it is notable that to be clinically relevant, sustained changes would likely be required that would extend into the sampled time interval of this study. Moreover, the possibility that neck pain participants exist who exhibit idiosyncratic responses cannot be excluded. To date, no such mechanisms have been measured and reported in the literature.

In conclusion, we found no significant change in blood flow in the posterior cerebrum or cerebellum in chronic neck pain participants after maximum head rotation and cervical manipulation. In addition, we found no clinically meaningful changes in the blood flow or velocity in the vertebral arteries before-after head positional change and spinal manipulation. Our study adds to a growing body of knowledge regarding the impact of head position and cervical manipulation on vascular and neural activity in patients with neck pain. It is the first study to measure cerebral blood flow, vertebral artery blood flow and velocity in patients undergoing neck manipulation for neck pain. Our study does not support the hypothesis that neck manipulation or neck rotation are associated with vasospasm of the vertebral artery.

\section{Patient and public involvement}

Patients and public were not involved in the development, design, recruitment and randomisation of this study.

\section{Author affiliations}

${ }^{1}$ Graduate Studies, Canadian Memorial Chiropractic College, Toronto, Ontario, Canada

${ }^{2}$ Faculty of Health Sciences, University of Ontario Institute of Technology, Oshawa, Ontario, Canada

${ }^{3}$ Radiology, McMaster University Faculty of Engineering, Hamilton, Ontario, Canada ${ }^{4}$ Faculty of Health Sciences, University of Ontario Institute of Technology, Oshawa, Ontario, Canada

${ }^{5}$ Faculty of Kinesiology and Physical Education, University of Toronto, Toronto, Ontario, Canada

${ }^{6}$ Department of Medicine, Division of Physical Medicine and Rehabilitation, University of Toronto, Toronto, Ontario, Canada

Acknowledgements Appreciation is extended to MRI technologists at the Imaging Research Centre for their assistance in data capture and for all of their support and effort with this study. Similarly, thanks are due to the Imaging Research Centre at St Joseph's Healthcare and Dr Shanker Nesathurai and the Department of Physical Medicine and Rehabilitation at St Joseph's Hospital for their ongoing support in this project. Special thanks are given to Dr Sheilah Hogg-Johnson for her timely help with this work's statistical analysis.

Contributors Concept development (provided idea for the research): NM, JT, GW and SM. Design (planned the methods to generate the results): NM, JT, MN and GW. Supervision (provided oversight, responsible for organisation and implementation, writing of the manuscript): NM, SM, JT, GW and PC. Data collection/processing (responsible for experiments, patient management, organisation or reporting data): NM, SM, MB and MN. Analysis/interpretation (responsible for statistical analysis, evaluation and presentation of the results): NM, MB, SM, JT, GW and MN. Literature search (performed the literature search): NM. Writing (responsible for writing a substantive part of the manuscript): NM and SM. Critical review (revised manuscript for intellectual content, this does not relate to spelling and grammar checking): NM, $\mathrm{SM}, \mathrm{PC}, \mathrm{MN}, \mathrm{GW}$ and JT. The corresponding author had full access to all the data in the study and had final responsibility for the decision to submit for publication.

Funding Partial financial support was provided by the Canadian Chiropractic Protective Association and NCMIC Research Foundation and Canadian Memorial Chiropractic College.

Disclaimer The funders of the study had no role in the study design, data collection, data analysis, data interpretation or writing of the report.

Competing interests Conflicts of interest were reported for this study include the following: JT is an occasional lecturer on behalf of NCMIC and CCPA, and MN received an honorarium for lecture on behalf of Bayer.

Patient consent for publication Not required.

Ethics approval The McMaster University Hamilton Integrated Research Ethics Board (HiREB) (REB\#1303) and Canadian Memorial Chiropractic College Research Ethics Board approved the study (REB\# $1604 \times 01$ ).

Provenance and peer review Not commissioned; externally peer reviewed.

Data sharing statement № additional data are available.

Open access This is an open access article distributed in accordance with the Creative Commons Attribution Non Commercial (CC BY-NC 4.0) license, which permits others to distribute, remix, adapt, build upon this work non-commercially, and license their derivative works on different terms, provided the original work is properly cited, appropriate credit is given, any changes made indicated, and the use is non-commercial. See: http://creativecommons.org/licenses/by-nc/4.0/.

\section{REFERENCES}

1. Albuquerque FC, Hu YC, Dashti SR, et al. Craniocervical arterial dissections as sequelae of chiropractic manipulation: patterns of injury and management. J Neurosurg 2011;115:1197-205. 
2. Ernst E. Manipulation of the cervical spine: a systematic review of case reports of serious adverse events, 1995-2001. Med J Aust 2002;176:376-80.

3. Norris JW, Beletsky V, Nadareishvili ZG. Sudden neck movement and cervical artery dissection. The Canadian Stroke Consortium. CMAJ 2000;163:38-40.

4. Cassidy JD, Boyle E, Côté P, et al. Risk of vertebrobasilar stroke and chiropractic care: results of a population-based case-control and case-crossover study. Spine 2008;33(4 Suppl):S176-83.

5. Boyle E, Côté P, Grier AR, et al. Examining vertebrobasilar artery stroke in two Canadian provinces. Spine 2008;33(4 Suppl):S170-5.

6. Côté $P$, Wong JJ, Sutton $D$, et al. Management of neck pain and associated disorders: A clinical practice guideline from the Ontario Protocol for Traffic Injury Management (OPTIMa) Collaboration. Eur Spine J 2016;25:2000-22.

7. Kjaer P, Kongsted A, Hartvigsen J, et al. National clinical guidelines for non-surgical treatment of patients with recent onset neck pain or cervical radiculopathy. Eur Spine J 2017;26:2242-57.

8. Hurwitz EL, Coulter ID, Adams AH, et al. Use of chiropractic services from 1985 through 1991 in the United States and Canada. Am J Public Health 1998;88:771-6.

9. Hurwitz EL, Chiang LM. A comparative analysis of chiropractic and general practitioner patients in North America: findings from the joint Canada/United States Survey of Health, 2002-03. BMC Health Serv Res 2006;6:49.

10. Haldeman S, Carey P, Townsend M, et al. Arterial dissections following cervical manipulation: the chiropractic experience. CMAJ 2001:165:905-6.

11. Patijn J. Complications in manual medicine: A review of the literature. J Manual Med 1991;6:89-92.

12. Smith RA, Estridge MN. Neurological complications of head and neck manipulations: A report of two cases. JAMA 1962;182:528-31.

13. Frisoni GB, Anzola GP. Vertebrobasilar ischemia after neck motion. Stroke 1991;22:1452-60.

14. Mann T, Refshauge KM. Causes of complications from cervical spine manipulation. Aust J Physiother 2001;47:255-66.

15. Schmitt HP. Anatomical structure of the cervical spine with reference to pathology of manipulation complications. Man Med 1991;6:93-101.

16. Mitchell J. Vertebral Artery Blood flow Velocity Changes Associated with Cervical Spine rotation: A Meta-Analysis of the Evidence with implications for Professional Practice. J Man Manip Ther 2009;17:46-57.

17. Kawchuk GN, Jhangri GS, Hurwitz EL, et al. The relation between the spatial distribution of vertebral artery compromise and exposure to cervical manipulation. J Neurol 2008;255:371-7.

18. Herzog W, Leonard TR, Symons B, et al. Vertebral artery strains during high-speed, low amplitude cervical spinal manipulation. $J$ Electromyogr Kinesiol 2012;22:740-6.

19. Dunne JW, Conacher GN, Khangure M, et al. Dissecting aneurysms of the vertebral arteries following cervical manipulation: a case report. J Neurol Neurosurg Psychiatry 1987;50:349-53.

20. Arnold C, Bourassa R, Langer T, et al. Doppler studies evaluating the effect of a physical therapy screening protocol on vertebral artery blood flow. Man Ther 2004;9:13-21.

21 Licht PB, Christensen HW, Højgaard P, et al. Vertebral artery flow and spinal manipulation: a randomized, controlled and observer-blinded study. J Manipulative Physiol Ther 1998;21:141-4.

22. Mitchell JA. Changes in vertebral artery blood flow following normal rotation of the cervical spine. J Manipulative Physiol Ther 2003;26:347-51.

23. Mitchell J, Keene D, Dyson C, et al. Is cervical spine rotation, as used in the standard vertebrobasilar insufficiency test, associated with a measureable change in intracranial vertebral artery blood flow? Man Ther 2004;9:220-7.

24. Mitchell J, Kramschuster K. Real-time ultrasound measurements of changes in suboccipital vertebral artery diameter and blood flow velocity associated with cervical spine rotation. Physiother Res Int 2008;13:241-54.

25. Rivett DA, Sharples KJ, Milburn PD. Effect of premanipulative tests on vertebral artery and internal carotid artery blood flow: a pilot study. J Manipulative Physiol Ther 1999;22:368-75.

26. Rivett DA, Sharples KJ, Milburn PD. Reliability of ultrasonograpahic measurement of vertebral artery blood flow. NZ J Physiother 2003:31:119-28.

27. Rossitti S, Volkmann R, Lofgren J. Changes of blood flow velocity in the vertebro-basilar circulation during rotation of the head in the normal human. Biomech Sem 1992;6:92-9.

28. Stevens A. Functional Doppler sonography of the vertebral artery and some considerations about manual techniques. Man Med 1991;6:102-5.
29. Quesnele JJ, Triano JJ, Noseworthy MD, et al. Changes in vertebral artery blood flow following various head positions and cervical spine manipulation. $J$ Manipulative Physiol Ther 2014;37:22-31.

30. Erhardt JW, Windsor BA, Kerry R, et al. The immediate effect of atlanto-axial high velocity thrust techniques on blood flow in the vertebral artery: A randomized controlled trial. Man Ther 2015;20:614-22.

31. Bakhtadze MA, Vernon $\mathrm{H}$, Karalkin AV, et al. Cerebral perfusion in patients with chronic neck and upper back pain: preliminary observations. J Manipulative Physiol Ther 2012;35:76-85.

32. Barrett KE, Ganong WF. Ganong's Review of Medical Physiology. 25th ed. New York, NY: McGraw-Hill Medical, 2015.

33. Guzman J, Haldeman S, Carroll LJ, et al. Bone and Joint Decade 2000-2010 Task Force on Neck Pain and Its Associated Disorders. Clinical practice implications of the Bone and Joint Decade 20002010 Task Force on Neck Pain and Its Associated Disorders: from concepts and findings to recommendations. Spine 2008;33(4 Suppl):S199-213.

34. Cambridge ED, Triano JJ, Ross JK, et al. Comparison of force development strategies of spinal manipulation used for thoracic pain. Man Ther 2012;17:241-5.

35. Descarreaux M, Dugas C, Raymond J, et al. Kinetic analysis of expertise in spinal manipulative therapy using an instrumented manikin. J Chiropr Med 2005;4:53-60.

36. Cohen E, Triano JJ, McGregor M, et al. Biomechanical performance of spinal manipulation therapy by newly trained vs. practicing providers: does experience transfer to unfamiliar procedures? Manipulative Physiol Ther 1995;18:347-52.

37. Triano JJ, Rogers CM, Combs S, et al. Developing skilled performance of lumbar spine manipulation. J Manipulative Physiol Ther 2002;25:353-61.

38. Triano JJ, Rogers CM, Combs S, et al. Quantitative feedback versus standard training for cervical and thoracic manipulation. $J$ Manipulative Physiol Ther 2003;26:131-8.

39. Ho SS, Chan YL, Yeung DK, et al. Blood flow volume quantification of cerebral ischemia: comparison of three noninvasive imaging techniques of carotid and vertebral arteries. AJR Am J Roentgenol 2002:178:551-6.

40. Lotz J, Meier C, Leppert A, et al. Cardiovascular flow measurement with phase-contrast MR imaging: basic facts and implementation. Radiographics 2002;22:651-71.

41. Ioannidis JP, Evans SJ, Gøtzsche PC, et al. Better reporting of harms in randomized trials: an extension of the CONSORT statement. Ann Intern Med 2004;141:781-8.

42. Parkes LM, Rashid W, Chard DT, et al. Normal cerebral perfusion measurements using arterial spin labeling: reproducibility, stability, and age and gender effects. Magn Reson Med 2004;51:736-43.

43. Cohen J. Statistical power analysis for the behavioral sciences. 2nd ed. New Jersey: Lawrence Erlbaum Associates, Publishers, 1988.

44. Cox RW. AFNI: software for analysis and visualization of functional magnetic resonance neuroimages. Comput Biomed Res 1996;29:162-73.

45. Holmes CJ, Hoge R, Collins L, et al. Enhancement of MR images using registration for signal averaging. J Comput Assist Tomogr 1998;22:324-33.

46. Jang JH, Kim JH, Jung $\mathrm{WH}$, et al. Functional connectivity in frontosubcortical circuitry during the resting state in obsessive-compulsive disorder. Neurosci Lett 2010;474:158-62.

47. Wang J, Wang L, Zang Y, et al. Parcellation-dependent small-world brain functional networks: a resting-state fMRI study. Hum Brain Mapp 2009;30:1511-23.

48. Fleiss JL. The Design and Analysis of Clinical Experiments. Toronto: Wiley, 1986.

49. Yurdakul M, Tola M. Doppler criteria for identifying proximal vertebral artery stenosis of 50\% or more. J Ultrasound Med 2011;30:163-8.

50. Bendick PJ, Glover JL. Vertebrobasilar insufficiency: evaluation by quantitative duplex flow measurements. A preliminary report. J Vasc Surg 1987;5:594-600.

51. Seidel E, Eicke BM, Tettenborn B, et al. Reference values for vertebral artery flow volume by duplex sonography in young and elderly adults. Stroke 1999;30:2692-6.

52. Arnetoli G, Amadori A, Stefani P, et al. Sonography of vertebral arteries in De Kleyn's position in subjects and in patients with vertebrobasilar transient ischemic attacks. Angiology 1989:40:716-20.

53. Sakaguchi M, Kitagawa K, Hougaku H, et al. Mechanical compression of the extracranial vertebral artery during neck rotation. Neurology 2003;61:845-7.

54. Debette S, Leys D. Cervical-artery dissections: predisposing factors, diagnosis, and outcome. Lancet Neurol 2009;8:668-78. 
55. Senter HJ, Sarwar M. Nontraumatic dissecting aneurysm of the vertebral artery. Case report. J Neurosurg 1982;56:128-30.

56. Sherman DG, Hart RG, Easton JD. Abrupt change in head position and cerebral infarction. Stroke 1981;12:2-6.

57. Haldeman S, Kohlbeck FJ, McGregor M. Unpredictability of cerebrovascular ischemia associated with cervical spine manipulation therapy: a review of sixty-four cases after cervical spine manipulation. Spine 2002;27:49-55.

58. Josien E. Extracranial vertebral artery dissection: nine cases. $J$ Neurol 1992;239:327-30.

59. Okawara S, Nibbelink D. Vertebral artery occlusion following hyperextension and rotation of the head. Stroke 1974;5:640-2.

60. Heinen SJ, Keller EL. The function of the cerebellar uvula in monkey during optokinetic and pursuit eye movements: single-unit responses and lesion effects. Exp Brain Res 1996;110:1-14.

61. Kanwisher N, Yovel G. The fusiform face area: a cortical region specialized for the perception of faces. Philos Trans $R$ Soc Lond $B$ Biol Sci 2006;361:2109-28.
62. Lamm C, Windischberger C, Leodolter U, et al. Evidence for premotor cortex activity during dynamic visuospatial imagery from single-trial functional magnetic resonance imaging and event-related slow cortical potentials. Neuroimage 2001:14:268-83.

63. Cavanna AE, Trimble MR. The precuneus: a review of its functional anatomy and behavioural correlates. Brain 2006;129:564-83.

64. Sengelhoff C, Nebelsieck J, Nassenstein I, et al. Neurosonographical follow-up in patients with spontaneous cervical artery dissection. Neurol Res 2008;30:687-9.

65. Dittrich R, Dziewas R, Ritter MA, et al. Negative ultrasound findings in patients with cervical artery dissection. Negative ultrasound in CAD. J Neurol 2006;253:424-33.

66. Nebelsieck J, Sengelhoff C, Nassenstein I, et al. Sensitivity of neurovascular ultrasound for the detection of spontaneous cervical artery dissection. J Clin Neurosci 2009;16:79-82.

67. Oktar SO, Yücel C, Karaosmanoglu D, et al. Blood-flow volume quantification in internal carotid and vertebral arteries: comparison of 3 different ultrasound techniques with phase-contrast MR imaging. AJNR Am J Neuroradiol 2006;27:363-9. 